Supporting Information for

\title{
In Situ Observation of a Photodegradation- induced Blueshift in Perovskite Nanocrystals Using Single-particle Spectroscopy Combined with Atomic Force Microscopy
}

Yoshua Albert Darmawan, Mitsuaki Yamauchi, Sadahiro Masuo*

Department of Applied Chemistry for Environment, Kwansei

Gakuin University, 2-1 Gakuen, Sanda, Hyogo 669-1337, Japan 
Evaluation of photodegradation processes of a single PNC with AFM measurement

In the main text, we showed one example of the evaluation of photodegradation processes of a single PNC with AFM measurement. Here, two additional results on the evaluation of photodegradation processes of a single PNC with AFM measurements are shown as Figures S1 and S2. 

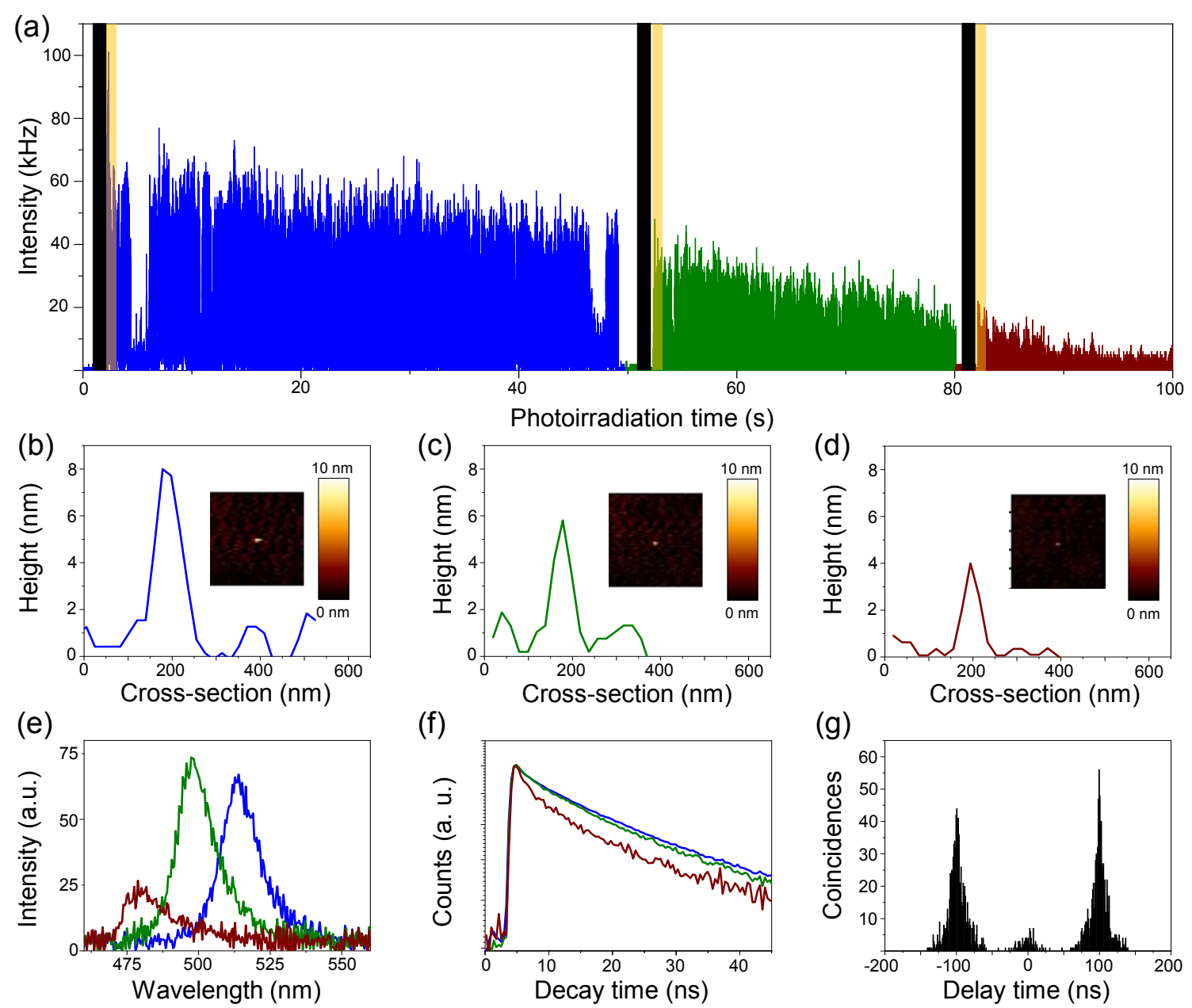

Figure S1 (a) PL time trace of a single PNC upon continuous photoirradiation. AFM images and PL spectra were measured at appropriate times indicated by black and yellow markers, respectively. (b-d) AFM images and cross-sections of the single PNC observed before photoirradiation (b), after $50 \mathrm{~s}$ photoirradiation (c), and after another $80 \mathrm{~s}$ photoirradiation (d). (e) PL spectra measured before continuous photoirradiation (blue), after $100 \mathrm{~s}$ photoirradiation (green), and after another $120 \mathrm{~s}$ photoirradiation (red). These spectra were measured by $5 \mathrm{~s}$ exposure time. (f) PL decay curves of the single PNC 
detected from blue (blue line), green (green line), and red regions (red line) in Figure S1

(a). (g) Photon correlation histogram of the single PNC detected from the blue region in Figure S1 (a). 
(a)

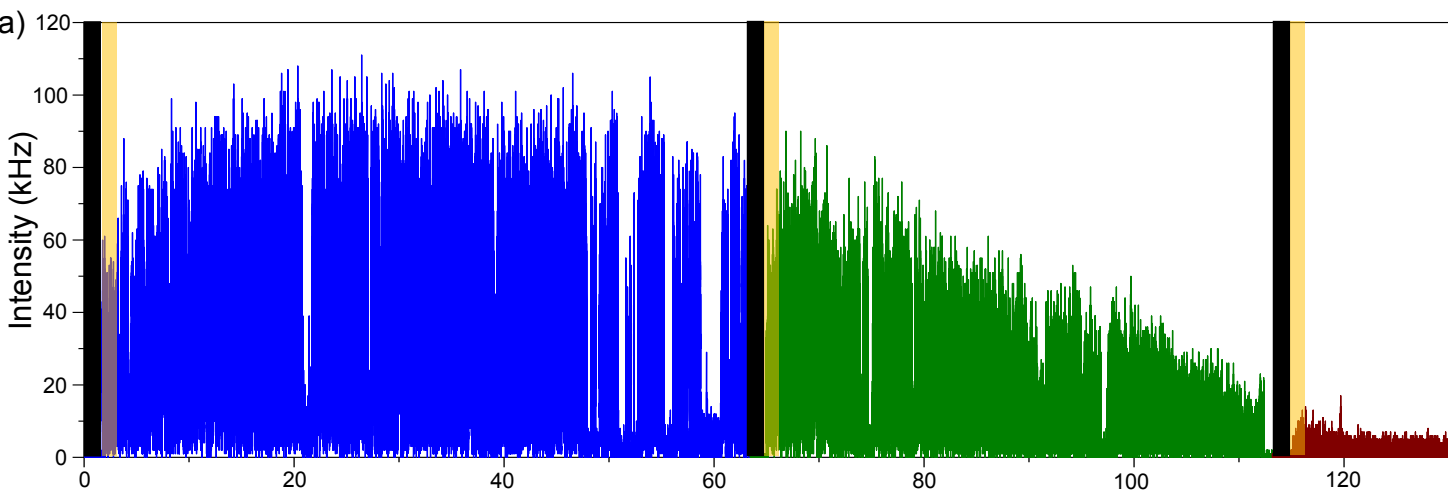

(b)



(e)

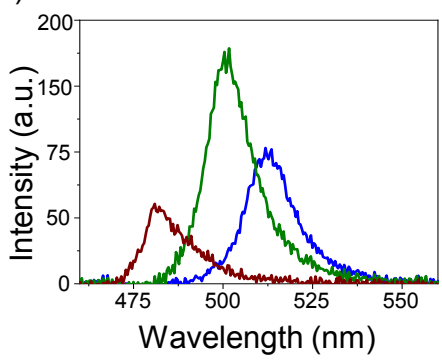

Photoirradiation time (s)
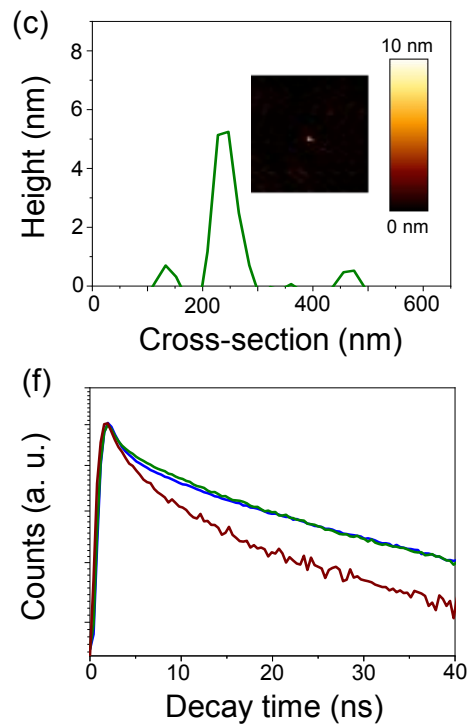

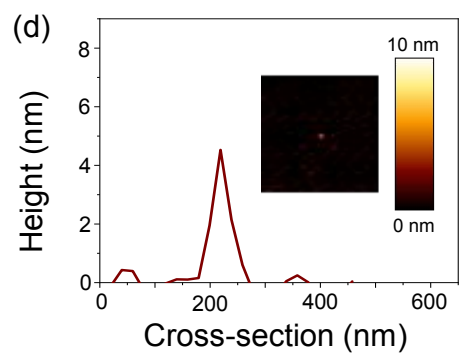

(g)

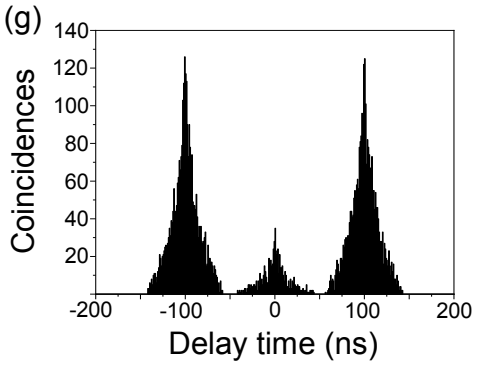

Figure S2 (a) PL time trace of a single PNC upon continuous photoirradiation. AFM images and PL spectra were measured at appropriate times indicated by black and yellow markers, respectively. (b-d) AFM images and cross-sections of the single PNC observed before photoirradiation (b), after $65 \mathrm{~s}$ photoirradiation (c), and after another $110 \mathrm{~s}$ photoirradiation (d). (e) PL spectra measured before continuous photoirradiation (blue), after $100 \mathrm{~s}$ photoirradiation (green), and after another $120 \mathrm{~s}$ photoirradiation (red). These spectra were measured by $5 \mathrm{~s}$ exposure time. (f) PL decay curves of the single PNC 
detected from blue (blue line), green (green line), and red regions (red line) in Figure S2

(a). (g) Photon correlation histogram of the single PNC detected from the blue region in Figure S2 (a). 